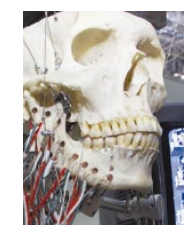

Profit potential

Japanese researchers set to gain as patent restrictions are lifted p504

\title{
Delays allowed foot-and-mouth epidemic to sweep across Britain
}

Jim Giles, London

Early delays in tackling food-and-mouth disease undermined the British government's attempts to control the outbreak, according to three epidemiological studies. All of the studies find that the epidemic is now out of control in the United Kingdom.

Animals at a farm near Newcastle in the north of England were almost certainly infected with the disease in early February, but the outbreak was not detected until sheep were moved over 400 kilometres to an abattoir in Essex. By the time full restrictions on the movement of animals were imposed on 23 February, transport of animals infected at the farm and abattoir had spread the disease across the country.

Teams from Imperial College, London, the University of Edinburgh and the government's Veterinary Laboratories Agency (VLA) in Surrey conducted separate studies on the basis of available government data.

"We're still in the exponential stage of the outbreak," says Mark Woolhouse, an epidemiologist at the University of Edinburgh (see Commentary on page 515).

The three studies make different predic-

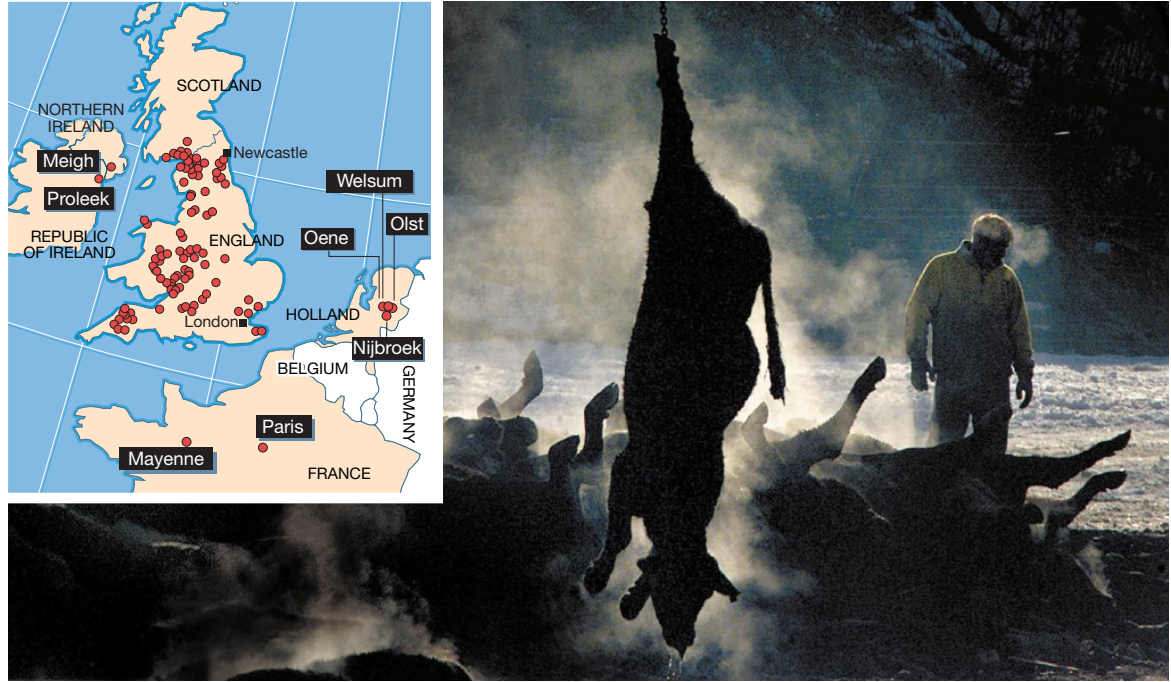

Out of control: culling is on the increase as foot-and-mouth spreads out across Britain — and Europe.

tions for the epidemic's development in the medium term. The VLA offers the most pessimistic scenario, projecting 4,411 cases by the end of June. At the other end of scale, the Edinburgh team estimates that 918 cases will be recorded by July - 627 cases had been

\section{Americans perplexed by GM food}

Corie Lok, Washington

More than half of Americans say they do not want to eat genetically modified (GM) food, but only one-fifth realize that they are consuming it already. These are some of the findings of a survey by a group set up to encourage a better-informed debate on the issue of transgenic foods.

A quarter of the people surveyed believe that GM foods are unsafe, and slightly more $(29 \%)$ consider them safe. The remainder are not sure either way, according to a telephone survey of 1,000 adults, performed for the newly launched Pew Initiative on Food and Biotechnology, based in Washington.

However, after being told that more than half of grocery store products in the United States already include GM foods, the percentage of respondents believing the foods to be safe jumped from 29 to 48 .

The survey results "suggest that consumer opinions about safety are not strongly held and may in fact be up for grabs", says Mike Rodemeyer, executive director of the Pew Initiative, a \$12 million public information project supported by the Pew Charitable Trusts.

This change of opinion in response to information about GM foods shows that "mandatory labelling and safety testing will go a long way to improving people's opinions about food biotechnology", says Jane Rissler of the Union of Concerned Scientists, which supports such labelling. The Biotechnology Industry Organization, a trade association, declined to comment on the survey results.

http://www.pewagbiotech.org

A () 2001 Macmillan Magazines Ltd confirmed by 27 March. Neither team was able to say when they thought the disease would be wiped out.

The government is now trying to limit the spread of the disease by culling all animals within 3 kilometres of infected premises in areas where the virus is widespread - a move that may result in the slaughter of some 600,000 animals.

Far smaller outbreaks have been confirmed in the Republic of Ireland, France and the Netherlands. Dutch vets have got permission from the European Union to vaccinate all animals in a 10-kilometre ring around the outbreaks, although the Dutch government says it will vaccinate only if it cannot slaughter all the infected animals quickly enough.

Despite calling in the Army to help dispose of culled animals, Britain now faces exactly such a backlog, leading some epidemiologists to suggest that vaccination might be useful. It had previously been ruled out because many countries will not accept meat imports from vaccinated areas.

Epidemiologists instead suggest cutting the interval between identifying and slaughtering infected animals. The gap averaged two to three days at the start of the epidemic, but the Imperial group says reducing this by a day could almost halve the total number of cases. 